\title{
Research on Moving Object Recognition and Tracking Based on Embedded Platform
}

\author{
Hengxiang $\mathrm{He}^{1, \mathrm{a}}$, Chunyu Chen ${ }^{2, \mathrm{~b}}$ and Yulong Qiao ${ }^{3, \mathrm{c}}$ \\ ${ }^{1}$ School of Information and Communication Engineering, Harbin Engineering University, Harbin \\ 150001, China \\ ${ }^{2}$ School of Information and Communication Engineering, Harbin Engineering University, Harbin \\ 150001, China \\ ${ }^{3}$ School of Information and Communication Engineering, Harbin Engineering University, Harbin \\ 150001, China \\ ahehengxiang0@163.com, bspringrain@hrbeu.edu.cn, 'cqiaoyulong@hrbeu.edu.cn
}

\begin{abstract}
Keywords: Moving target tracking ; Kalman filter ; DM642
\end{abstract}
For the rest of the paper, please use Times Roman (Times New Roman) 12

Abstract. In recent years, the concept of unmanned technology and automatic monitoring based on AI has gradually become a hotspot, which all need the equipment to identify the target in video image quickly and timely.This paper designs a set of video moving target recognition and tracking system based on embedded platform, uses Ti's latest 6000 series DSP to process the video data acquired by the High-speed camera, automatically identifies the moving target in the video screen, then tracks and predicts its movement trajectory.

\section{Introduction}

In computer vision field,moving object detection is one of the key steps in information extraction ,which is an important problem in the field of modern image processing ${ }^{[1]}$. In this paper, the detection process for the moving object is carried out in a static background, which means, the view of the camera is stationary (the camera and the background are fixed), only the monitored target is moving.At present, there are three main methods of moving target detection, namely, background subtraction, inter-frame difference and optical flow .

When the video tracking system starts to work, there is a lot of noise in the real-time picture captured by the camera ${ }^{[1]}$. By using the appropriate algorithm, it can reduce the interference of noise, so the system can provide accurate information including the exact location and speed of the tracking target and so on. By using the corresponding tracking algorithm can quickly and accurately predict the location and speed at the next moment, this feature is great benefit to maintain the stability and continuity of tracking, even if the target can not be detected at a short time, the system can also keep as continuous and accurate as possible ${ }^{[2]}$. At present, the most common tracking algorithms are Kalman filter, Condensation, Mean Shift and particle filter.

The basic idea of the Kalman filter tracking algorithm is to use the unbiased minimum variance criterion to predict the state of the moving target at the follow-up time by the state transition equation of the current state of motion. Kalman filter in the prediction with unbiased ${ }^{[3]}$, stable and optimal advantages, its real-time and anti-noise is also excellent, it is a good filter out the Gaussian noise.

Compared with the above-mentioned several common filters, it can be found that the Kalman filter algorithm is relatively mature and real-time is high. Therefore, the tracking algorithm based on Kalman filter is used to track the moving target.

In this paper, firstly , the basic principle and implementation method of Kalman filter are introduced. Then, the method is applied to video moving target recognition and tracking. Finally, the effectiveness and performance of the algorithm are verified by simulation experiments. 


\section{Kalman Filter}

The tracking of the moving target rely on the existed data, such as the coordinate and speed of the previous time, using the appropriate tracking algorithm to predict the coordinates, velocity and other data at the next moment. This design uses the Kalman filter algorithm to track the moving target ${ }^{[4]}$.

Kalman filter has the following two characteristics:

1) describe its mathematical formula with state space;

2) solution is recursive and can be applied without modification to a smooth and non-stationary environment.

That is, the estimated value of the current time can be obtained by only relying on the estimated value of the previous time and the observed value at the current time. It is not necessary to record observations or estimates of all previous times, which greatly simplifies the implementation of the Kalman filter. ${ }^{[5]}$

The main idea of Kalman filter is to predict and correct in time series. These two steps are iteratively updated. That is to say, the predicted value is calculated first, then the new information obtained from the observed value and the gain of the Kalman filter are used to fix the current predictive value. In this way, the prediction value can be obtained from the optimal value, the optimal value from the predicted value, and the interaction between the optimal value and the predicted value, so as to realize the real-time processing.

\section{Video Moving Target Recognition and Tracking}

The video moving target recognition means acquiring the picture through the camera, the processor processing and analyzing the collected picture data, identifying and marking the target in the camera field of view, extracting the coordinates and then sending it to other processing modules for analysis, such as the Kalman filter Device. After the Kalman filter processing and analysis, the predicted value of the target trajectory at the next moment can be obtained, and the system makes a pre-judgment in advance.Even if the target disappears within a short period of time, system also can be normal and consistent.

This paper designs the background subtraction as the main method, but taking into account the different time, the background brightness will change, so combined with the some features of inter-frame difference, the background will be regularly refreshed.

\section{Video Single Target Recognition}

Whether it is single-target or multi-target, the premise is to filter out the moving objects at the video screen, then determine the number of targets and coordinates. In this paper, the background subtraction is used to identify the moving target. When the camera shots a frame, the DM642 processor will read the data of this frame and count the number of frames that have been processed.

Considering when the device is powered on, the picture captured by the camera may jitter, it will have a greater impact on the follow-up recognition, after several actual tests, taking into account the system stability, boot time and other factors, finally, set the third frame as the background frame.

In actually, changes on time or weather will have a great impact on the ambient light, if the background frame remains the same, with the delay, the system noise will gradually increase until the submerged target signal. In orde to solve this problem, this paper chooses to combine some characteristics of the interframe difference,so refreshes the background frame every 5 minutes.

\section{Multi-target Video Recognition}

Multi-target and single-target recognition are the same in moving target detection, but target quantity detection and the determination of the coordinates are different in the subsequent. In the 
target quantity and centroid recognition program, the multi-target recognition algorithm is more complex. In the actual design, the principle of the two is different.

As mentioned in the previous section, in order to facilitate the user to visually see the moving target, after the completion of the mobile target detection, pixels undetected moving will be set to 190 , that is displayed as white, and pixels detected moving will be set to 0 , that is displayed as black. However, in the multi-target recognition program, in order to facilitate the follow-up algorithm, need to set array of 190 pixels to 0 , set the original 0 to 1 . Then get a new $360 * 288$ size of the array, which represents each line has 360 pixels, each column has 288 pixels, the reason of the first value of each pixel set to 0 or 1 , is to facilitate the follow-up statistics existence of a moving target on the pixels of each row and each column in $t$ is het.

In the case of an example, each line has 360 pixels, a total of 288 lines, the program will add up to 360 pixels per line, and its sum is copied to a corresponding size of 288 array. Then the program begins to analyze the size of the 288 array, if the value is 0 , which means there is no moving target exists on the corresponding line, if not 0 , there is a moving target. The program determines that there are several sections in the array that are consecutive non-zero, which represents several moving targets in the row target, then get the center coordinates and length of each block. Similarly, use this method to find the column target has several moving targets, and extract the relevant data. It is assumed that the number of rows is 2 and the number of columns is 2 , which is theoretically 2 to 4 of the moving target in the video screen. To further determine the target number and the target coordinate, extract the coordinates of the $2 * 2$, the program finds the pixel that corresponds to the position of the coordinate, determines whether it is 1 , if 1 , the target exists, then mark the target with a rectangle, otherwise, it does not have a target.

\section{Video Target Tracking}

After coding the program, DM642 processed coordinates were substituted into the Kalman filter, Kalman filter process and predict the data to remove the Gaussian noise in the signal and get the prediction data of the next moment in order to make the target continuous tracking.

Due to the multi-target recognition program in real-time aspects need to be optimized, only 5 frames per second, but single-target recognition program is better, so only use the Kalman prediction program to single-target recognition .

\section{Experimental Analysis}

In this paper, use MATLAB to generate multi-target trajectories and add Gaussian white noise to the trajectories of each target to simulate the interference received by the actual detector and generate several Gaussian white noise as process noise and measurement noise, finally encapsulate the data according to a certain format and save it as a .DAT file.

In MATLAB, the design of the preparation of kalman filter program to verify the feasibility of the filter, and compare the target real trajectory and filter generated prediction trajectory to determine the effect. The specific process shown in Fig.1:

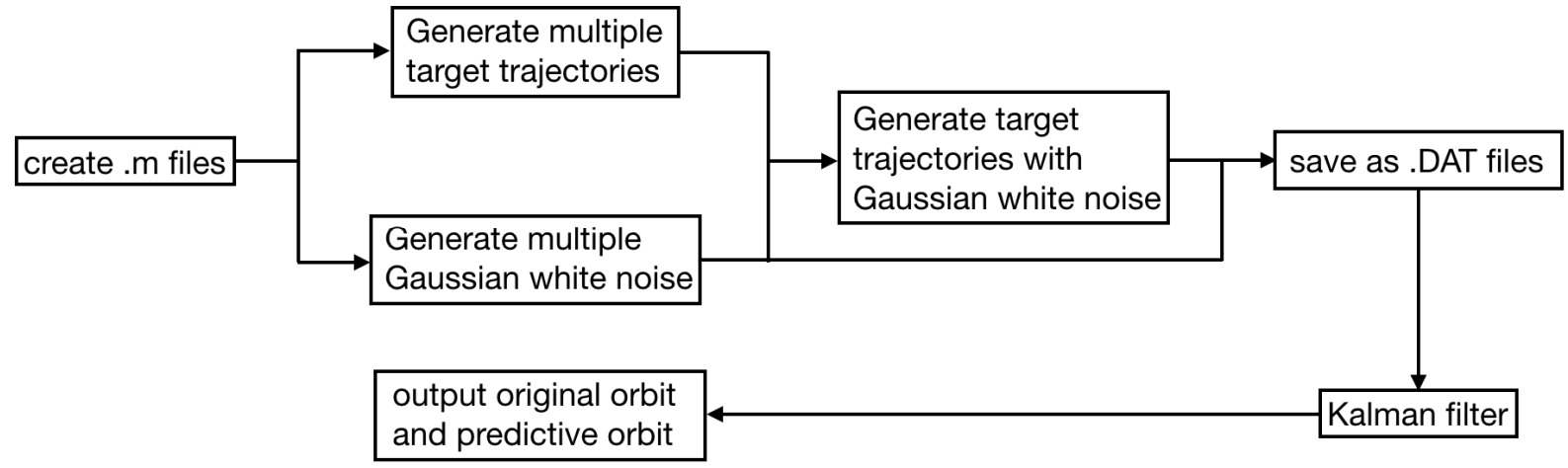

Fig.1 Implementation of the Kalman filter 
When the Gaussian white noise variance take 100, do the same experiment, the simulation results shown in Fig.2.

In the process of video target recognition, it is necessary to compare the value of the pixel position between the background frame and the real-time frame to determine whether the change has occurred at the point, the size of the threshold is a key factor. If the threshold is too high, the target will be lost easily. If the threshold is too small, the noise will be too much. After several tests, it was found that when the threshold was set between 30 and 35, the noise was better filtered and had little effect on the recognition of target recognition. When the threshold is 30, the simulation results are shown in Fig.3.

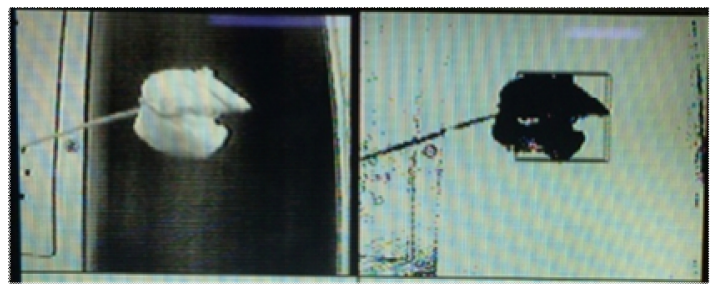

Fig. 2 simulation results(threshold is 30 )

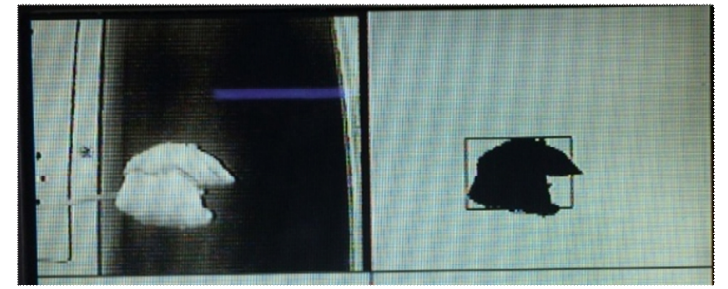

Fig.3 Simulation results after noise reductio

After selecting the appropriate threshold, there is still some noise in the picture from the simulation results. In this paper, the following method is used to reduce the noise: the system will save the detected picture to a $360 * 288$ size array, If the point is black, there is a moving target, then the point data of the right and the bottom of the point is read, if it is black, it is determined that the point is valid and not processed. If it is white, it is determined that the point is noise and the point is displayed as white. Then analyze the next point, followed by the cycle, until the last point, which is to keep the system does not overflow, the last row of pixels forced to white. Simulation results after noise reduction as shown in Fig.3, it can be seen that the noise is basically filtered and the moving target is well preserved.

The simulation results of the video target tracking are shown in Fig.5. The simulation results are concatenated with pictures of the four times. The photo in the upper left corner is the earliest and the photo in the lower right corner is the latest. At the same time, the upper left corner is the live view and the Kalman filter predicts the target next block diagram; the upper right corner is the moving target identification map, and the block diagram is used to indicate the moving target identified at the current time; The larger black dot indicates the predicted value obtained by the Kalman filter, the smaller black dot indicates the actual position of the moving target, and the lower right corner is the Kalman filter prediction error graph.

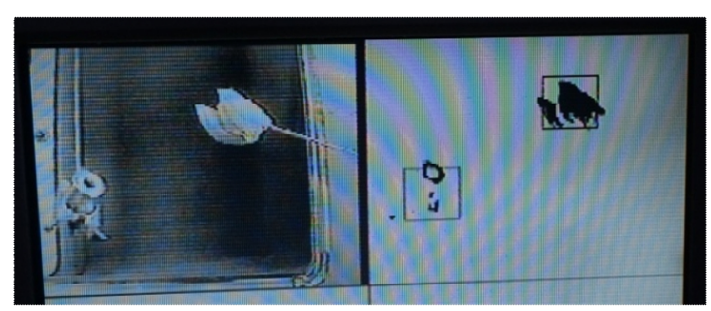

Fig.4 video multi-target recognition

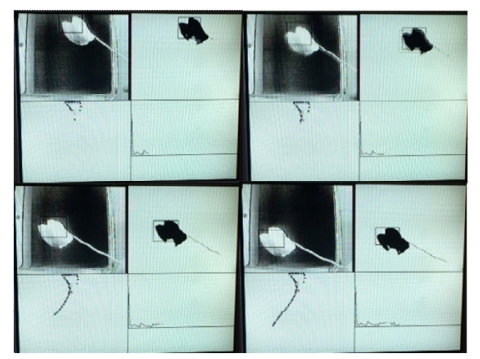

Fig.5 Video moving target tracking program renderings

The simulation results of video multi-target recognition are shown in Fig.4. It can be seen from the simulation results that the multi-target recognition algorithm can identify multiple moving targets in the field of view and can accurately identify centroid coordinates, length, width and other data, the program runs fast, handles a frame on average of $200 \mathrm{~ms}$ and displays at the screen.

It can be seen that the Kalman filter has a large error in the initial time, but the error is decreasing and the prediction is more and more accurate with the passage of time. The motion target recognition program is excellent for detecting moving objects in the field of view and to mark them with a rectangular box. The Kalman filter can efficiently obtain the next position prediction of the target and mark it in the upper left corner of the window. 


\section{Conclusion}

In this paper, a video target detection and tracking algorithm based on Kalman filter is designed by using DM642 development platform. It can be seen from the simulation experiment that the algorithm can effectively detect the target of motion in the video and track the target. The detection part of the algorithm proposed in this paper also applies to the situation of multi-motion target, while the tracking part only considers the situation of single target.

While detecting and tracking the target, the Kalman filter is used to predict the trajectory of the target. It can be seen from the simulation experiment that the Kalman filter modeled with variable acceleration linear motion has obvious advantages for the tracking of curve motion or other irregular motion, and its prediction is basically consistent with the target trajectory.

In the case of large signal-to-noise ratio, the Kalman filter can better track the target, but when the signal-to-noise ratio is small, its trajectory prediction ability is generally, and the noise covers the target trajectory.

The relative error of the Kalman filter's prediction is larger initially and then tends to be stabilized. When the noise variance is large, the fluctuation range is larger and the time of stabilization is longer.

\section{ACKNOWLEDGMENT}

The authors would like to thank the financial support from National Natural Science Foundation of China under Grant 61371175.

\section{References}

[1] Aghajan H, Cavallaro A.Multi-Camera Networks:Principles and Applications. Pittsburgh: Academic Press, 2009

[2] Jia Y.Alternative proofs for improved LMI representations for the analysis and the design of continuous-time systems with polytopic type uncertainty: a predictive approach.IEEE Trans Automat Contr, 2003, 48:1413-1416

[3] Snidaro L, Visentini L, Foresti G L.Intelligent Video Surveillance: Systems and Technology.Boca Raton:CRC Press, 2009.363-388

[4] Li B, Yan W.A sensor fusion framework using multiple particle filters for video-based navigation.IEEE Trans Intell Trans Syst, 2010, 11:348-358

[5] Denman S, Lamb T, Fookes C, et al.Multi-spectral fusion for surveillance systems.Comput Electr Eng, 2010, 36:643-663 [5] R.J. Ong, J.T. Dawley and P.G. Clem: submitted to Journal of Materials Research (2003) 